\section{Holistic Total Pain Management in Palliative Care: Cultural and Global Considerations}

\author{
Jeannine M. Brant, PhD, APRN, AOCN, FAAN
}

Billings Clinic, $280010^{\text {th }}$ Avenue North, Billings, MT 59101, USA

\section{ABSTRACT}

Pain is a significant symptom in patients with chronic and life-threatening illness. While pain is traditionally thought of as a physiological experience, total pain recognizes the interplay of psychological, cognitive, social, spiritual, and cultural factors that influence the pain perception and total experience. Comprehensive pain assessment and management are foundational goals within the scope of palliative care, and optimal management depends on addressing each domain of the total pain experience. An overview of the total pain experience is provided, and clinicians should consider psychological, cognitive, social, spiritual, and cultural aspects in assessing pain. Pain management also addresses all domains, and suggestions are provided which address pain management barriers and challenges. First, patients should be educated about the benefits of pain management and importance to adhere to the plan of care. Second, healthcare professionals need education in order to manage pain properly and should adhere to internationally recognized evidence-based guidelines to provide care. Third, barriers to overcome system issues need to be addressed, such as working with governments and Ministries of Health to increase opioid availability for those in need and to ensure that patients can have access to opioids whether in the hospital, home, city, or rural area. While pain is a complex phenomenon, a comprehensive management plan can alleviate suffering for patients and their families.

KEY WORDS: Total pain; Holistic; Palliative care; Culture; Opioid availability.

\section{INTRODUCTION}

Palliative care encompasses the physical, psychological, social, spiritual, and cultural domains of patients and their caregivers. ${ }^{1}$ Physical symptoms can be problematic when they occur as patients have difficulty focusing on other quality of life (QoL) issues. Pain is one of the most common and problematic symptoms that occurs in conjunction with chronic and advanced illness and requires specific attention. Incorporating comprehensive pain services into any palliative program is paramount. ${ }^{2}$ This review will address the multitude of culturally relevant challenges in implementing pain management services into a palliative care program. The holistic components of pain and their influences on the pain assessment and management plan, and pain assessment and management barriers will be discussed.

\section{TOTAL PAIN}

Total pain is a holistic experience that extends beyond the physiological domain and was first introduced by Dame Cicely Saunders in the 1960's. Total pain recognizes the holistic nature of pain and the interplay of psychological and social well-being, spirituality, and culture. Symptoms rarely occur in isolation; rather, they cluster with other symptoms and are influenced by the psychological, social, and cultural characteristics of the individual. These holistic aspects of pain are discussed in the following section.

\section{PHYSICAL PAIN}

According to the International Association for the Study of Pain, pain is "an unpleasant sen- 
sory and emotional experience associated with actual or potential tissue damage, or described in terms of such damage." While physical pain is the physiological component, this definition emphases the importance of the physical impact on the entire person. ${ }^{3}$ Other deleterious physical symptoms can occur in conjunction with pain. The most common of these is fatigue. ${ }^{4}$ Dyspnea, drowsiness, sleep disturbance, nausea, and loss of appetite are other physical symptoms that need to be assessed when an individual is experiencing pain..$^{5-7}$ Depression is a common problem that also co-occurs with pain. Current research is focusing on underlying. mechanisms that may be responsible for co-occurring symptoms, also known as symptom clusters. Most importantly, clinicians should recognize that pain may contribute to other symptoms; thereby, managing pain may eliminate other symptoms as well.

\section{PSYCHOLOGICAL PAIN}

Emotional distress, depression, anxiety, uncertainty, and hopelessness are all forms of psychological pain that can co-occur with physical pain with depression being one of the most common psychological symptoms. ${ }^{8}$ One systematic review indicated that the co-occurrence of pain and depression is approximately $36.5 \%$. The more intense the pain was, the more likely the individual was to be depressed $(p<0.05)$. Patients with depression may use more affective words to describe pain such as fearful. QoL has also been shown to be worse in those with both pain and depression. ${ }^{9}$ Depression is also found to be a significant barrier to manage pain, underscoring the importance of managing depression in order to improve pain. ${ }^{10}$

According to the National Comprehensive Cancer Network, distress is an "unpleasant experience of a mental, physical, social, or spiritual nature." It can affect the way an individual thinks, feels, or acts and can make coping more difficult. ${ }^{11}$ Distress should be screened for each patient visit. While pain can be a reason for the distress, other reasons should also be noted as all factors can increase the occurrence and severity of the pain experience. ${ }^{12,13}$ In one study, concurrent physical symptoms and psychosocial distress occurred in patients attending a cancer pain clinic compared to those who did not attend the pain clinic. ${ }^{14}$ Clinicians should recognize that pain and distress commonly co-occur.

\section{COGNITIVE-BEHAVIORAL INFLUENCES}

Cognitive-behavioral responses to pain are additional components of holistic total pain. One cognitive response could be the patient's failure to acknowledge the pain for fear that this represents progressive disease. Other patients may feel the need be tough and endure the pain. This cognitive denial of pain, which could be stemmed from cultural or spiritual beliefs, can interfere with optimal management. The cognitive-behavioral domain can also be positively used to address overall pain. Cognitive behavioral therapy that can be used to ameliorate pain in some patients includes building self-esteem, optimism, and mastery of pain control. ${ }^{15}$
Catastrophizing is another recognized cognitive trait associated with pain. Patients who exhibit this behavior ruminate or exaggerate their pain, and catastrophizing is commonly linked to depression. ${ }^{16}$ Cognitive-behavioral approaches should be considered in the overall pain management plan.

\section{SOCIAL INFLUENCES}

The social context of cancer pain is well-recognized. Pain can lead to social isolation, disengagement from meals and other activities, caregiver burden, and inability to afford analgesics to control the pain. Adequate social support is predictive of less distress, depression, and anxiety. ${ }^{17}$ The National Comprehensive Cancer Network (NCCN) Distress thermometer includes measurement related to social distress. Again, distress assessment should be incorporated into daily practice. When social distress is detected, psychosocial interventions, including education and coping-skills training may be useful adjuncts to medical management of pain.

\section{SPIRITUAL AND RELIGIOUS INFLUENCES}

Spirituality, defined as the need to be connected to a higher power, has a significant association with pain. Religion, on the other hand, includes the practices associated with an organized system. Spiritual and religious influences of pain may vary by religion and even by individual belief within a religion. For example, some patients may feel that God is punishing them, and that their reward in heaven will be greater if they endure pain. In the Muslim faith, some patients feel that pain is considered a punishment from God; however, Islamic teachings report differently. ${ }^{18}$ Spiritual and religious beliefs can therefore, be misperceived and influence how an individual perceives the pain and manages the pain. Often a religious leader or chaplain can explore spiritual and religious questions with patients individually, which can add to the overall pain management plan.

Hope is a concept commonly associated with spirituality and is an important component of most religious faiths. Studies reveal hope to be positively correlated with spiritual well-being $(p<0.01)$ and negatively correlated with average pain intensity $(p=0.02)$, worst pain intensity $(p<0.01)$, pain interference with function $(p<0.05)$, anxiety $(p<0.01)$, and depression $(p<0.01)$. Depression especially influenced this relationship, which reinforces the need to manage pain in a holistic manner. ${ }^{19}$

\section{CULTURAL INFLUENCES}

Pain expression is an individualized experience, which is influenced by culture or ethnicity. It can represent the individual's conceptual meaning of pain, pain perception, and coping abilities. One systematic review found that some ethnic groups expressed more severe pain. Asians tended to normalize pain whereas westerners were more likely to seek help for their pain. ${ }^{20}$ A second recent systematic review of 26 studies compared pain responses of African Americans (AA) to non-Hispanic Whites (NHW) and found AAs demonstrated lower pain tolerance. ${ }^{21}$ 
Another large meta-analysis of 22 studies found Asian patients to have more pain barriers compared to Western patients such as concerns about cancer progression, drug tolerance, fatalism, and pain management barriers. ${ }^{22}$ This could provide rationale for why Asians may try to normalize pain.

Overall, patients from some ethnic or cultural groups may have difficulty in communicating with their care-providers about pain. ${ }^{23}$ Providers as well may have barriers toward patients who are ethnically or racially different than themselves. For example, in one study, Western Caucasian physicians were noted to underestimate pain in $75 \%$ of AAs and $64 \%$ of Hispanics. ${ }^{24}$ These patients also reported suboptimal pain management. These overall differences underscore the need for a patient-centered approach for the management of pain.

\section{PAIN MANAGEMENT BARRIERS}

The holistic components of pain can strongly influence the individual pain experience. These influences can contribute to some of the major barriers that interfere with optimal pain management in many regions around the world. Pain assessment and management barriers commonly occur in most cultures and involve three levels: 1) patient and caregiver, 2) healthcare professional, and 3) systems. Sadly, these barriers have existed for more than 20 years and have not been adequately addressed.

For patients and caregivers, stoicism, failure to report pain, and fear about addiction are common barriers. For health care professionals, failure to consistently assess pain, lack of knowledge about pain management strategies, fears of addiction and beliefs that pain is an inevitable component of cancer are common problems. When supportive care services are available for pain and symptom management, many patients may not even know they exist, because physicians and other professionals may not consistently refer patients to these services. ${ }^{25,26}$ In regards to systems, opioids and other management options may not be fully available in some countries. Opioid use outside of Western societies is minuscule in most of the world (Figure 1). Tight regulations through the governmental agencies such as the Ministry of Health can be a barrier for optimal pain management. ${ }^{27}$ Additional efforts that address these barriers are imperative to achieve meaningful progress. ${ }^{28}$

\section{INTERVENTIONS TO ADDRESS BARRIERS}

Multiple efforts to address pain management barriers are occurring around the globe. Education for all stakeholders is important to overcome both knowledge and attitude barriers. Education, guideline adherence, medication coaching, and addressing

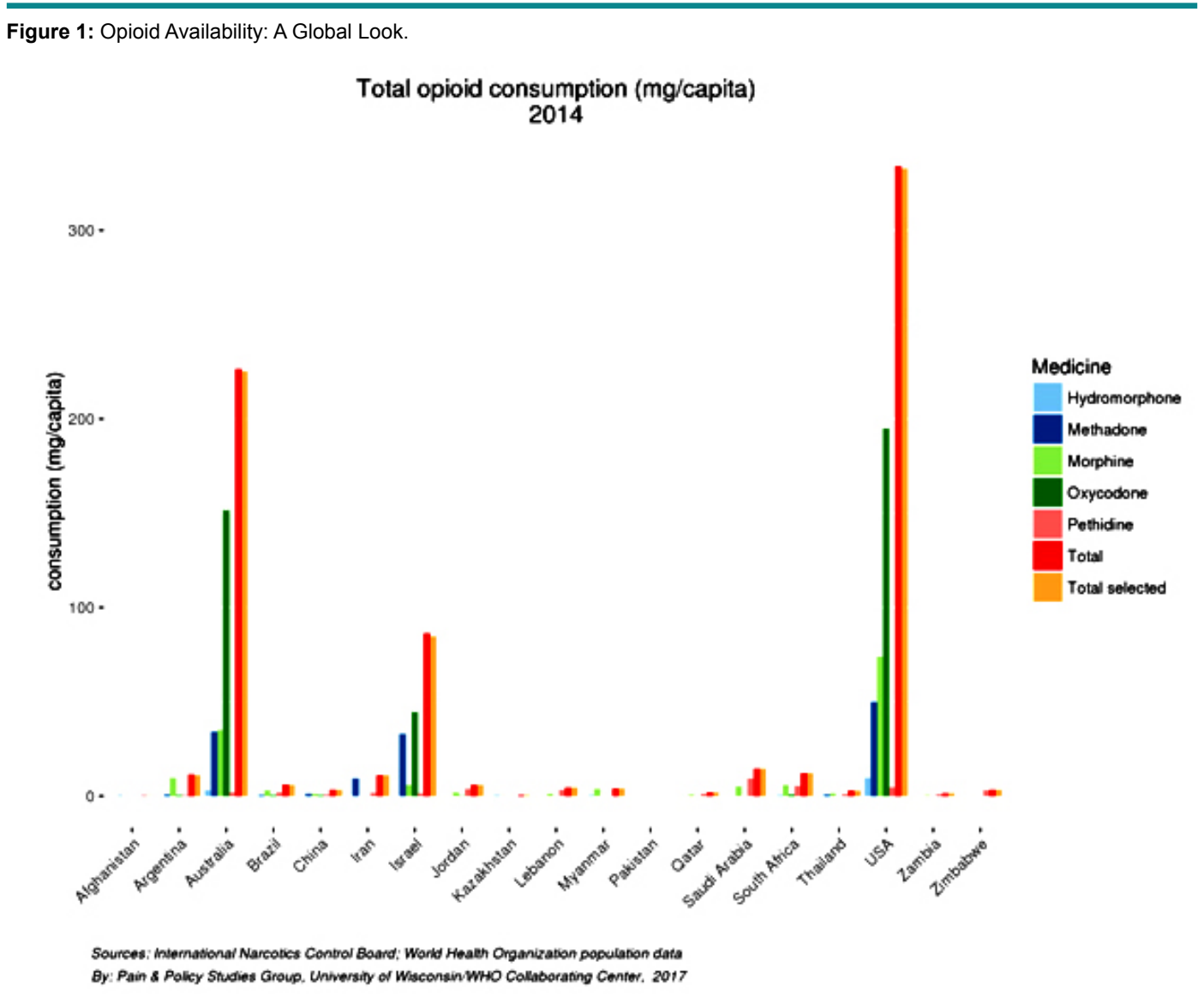


fears of addiction and substance use disorders are all potential solutions for improving pain management quality.

\section{PATIENTS AND CAREGIVERS}

\section{Educational Approaches}

Educational efforts are occurring worldwide. The Middle Eastern Cancer Consortium has played a role to provide pain management education throughout the Middle East for the past two decades. ${ }^{27,29}$ Palliative care education includes the management of pain has also been instrumental in that same region. ${ }^{2}$ Education includes conducting a comprehensive pain assessment and employing optimal management strategies for pain relief. Discussions should also ensue about belief systems regarding pain and fears of addiction which can assist to bridge the gap between suffering and comfort. Two systematic reviews (21 trials) and meta-analyses (15 trials included in one meta-analysis, 26 in another) found that education for patients, caregivers, and healthcare professionals can decrease pain intensity, ${ }^{30-32}$ and the greater the dose of the educational intervention, the better the pain outcomes. ${ }^{31}$ In regards to patient education, repeated faceto-face interactions seem to be the most effective compared to written information. ${ }^{33}$ When education is consistently delivered, sustained pain improvements have been demonstrated over time. Healthcare professionals should include pain education in daily care. ${ }^{32}$

Education plays an important role in the overall management of pain. More studies and educational models should be proposed to suggest how best to implement educational interventions within the scope of care and to determine the combina- tion of interventions that is most beneficial and cost-effective for patients and healthcare systems. ${ }^{34}$

\section{Analgesic Self-Management}

One of the biggest patient level barriers is not following the pain management plan of care. Both patients and caregivers can influence the self-management plan. Common reasons for not following the plan are fear of addiction, forgetfulness, and untoward side effects. Studies found that analgesic adherence ranges from $49 \%$ to $91 \%$ for long acting opioids and as low as $20 \%$ for as needed pro re nata (PRN) opioids. ${ }^{35-37}$ Depression and older age were found to be predictors of not following the pain management plan in one study. Additionally, patients were unsure about what their exact medication regimen was comprised of and therefore, it was not followed. ${ }^{36}$ Reasons for lack of self-management should be carefully assessed with patients and caregivers. Education about addiction, the importance of comfort, and clear instructions about the pain management plan should be provided. The overall message should not be paternalistic but rather coaching and collaborative. ${ }^{38}$

\section{HEALTH CARE PROFESSIONALS}

\section{Education}

Not believing the patient's report of pain is a significant barrier that can significantly impact the quality of pain management. ${ }^{39}$ Health care professionals should have a therapeutic relationship with the patient, and listen carefully to the patient's report of pain. While substance use disorders and addiction exist, the undermanagement of pain in palliative care patients is substantial

\begin{tabular}{|c|c|}
\hline Aberrant Behavior & Behaviors indicative of prescription drug abuse, some of which are more indicative of abuse or addiction. \\
\hline Abuse & Use of a drug for nontherapeutic purposes to obtain psychotropic effects. \\
\hline Addiction & $\begin{array}{l}\text { A primary, chronic, neurobiologic disease, with genetic, psychosocial, and environmental factors influencing its develop- } \\
\text { ment and manifestations. It is characterized by behaviors that include one or more of the following: impaired control over } \\
\text { drug use, compulsive use, continued use despite harm, and craving. }\end{array}$ \\
\hline Diversion & Unlawful channeling of pharmaceuticals from legal sources to the illegal marketplace. \\
\hline Illicit Substance & A substance that is not legally permitted or authorized. \\
\hline Misuse & Use of a prescription drug without a prescription or in a manner that is not prescribed. \\
\hline Narcotic & $\begin{array}{l}\text { An archaic term for an opioid analgesic; currently the term is used by law enforcement to describe illicit substances with a } \\
\text { potential for abuse such as heroin, cocaine, or methamphetamine. }\end{array}$ \\
\hline Opioid & $\begin{array}{l}\text { A medication that exerts its primary pharmacologic response by its binding to the opioid receptors in the central nervous } \\
\text { system (CNS). This term is preferred to the term "narcotic". }\end{array}$ \\
\hline Physical Dependence & $\begin{array}{l}\text { A state of adaptation manifested by a drug class specific withdrawal syndrome that can be produced by abrupt cessation, } \\
\text { rapid dose reduction, decreasing blood level of the drug, and/or administration of an antagonist. }\end{array}$ \\
\hline Pseudoaddiction & $\begin{array}{l}\text { Pattern of drug-seeking behavior in patients with pain who are receiving inadequate pain management; can be mistaken } \\
\text { for addiction. }\end{array}$ \\
\hline Tampering & Manipulating a pharmaceutical to change its drug delivery performance. \\
\hline Tolerance & $\begin{array}{l}\text { A state of adaptation in which exposure to a drug induces changes that result in a diminution of one or more of the drug's } \\
\text { effects over time. }\end{array}$ \\
\hline
\end{tabular}


and professionals should advocate for better pain relief in their regions. ${ }^{40}$ Understanding the differences between addiction, tolerance, and physical dependence, and understanding the differences of addiction and other high risk behaviors is the first step in overcoming this knowledge gap. A list of terms is included in Table $1 .^{3,41,42}$ When clinicians have this knowledge, they can then educate patients and caregivers which will decrease some of the overall fears of addiction, especially in palliative and end-oflife care. Education has been shown to improve attitudes about pain. ${ }^{43}$ Finally, education is important in improving health care professional knowledge and opioids, which opioids to prescribe, and co-analgesics that can improve overall comfort. Pain management is both an art and a science and requires specific education. Clinical guidelines are one way to educate clinicians and ensure that all patients receive consistent, quality pain management.

Clinical Practice Guideline Adherence. While a plethora of guidelines exist to assist clinicians in managing pain, ${ }^{41,44,45}$ studies reveal that only $22 \%$ to $45 \%$ of clinicians use a pain guideline. ${ }^{26,46}$ Some efforts are underway to encourage clinicians to use practice guidelines. In one study setting, nurse practitioners received weekly feedback on patient pain scores and how consistent their recommended interventions aligned with clinical guidelines. This audit and feedback intervention resulted in significantly less overall pain interference and interference with general activity and sleep. Satisfaction with pain relief increased significantly from $68.4 \%$ to $95.1 \%{ }^{47}$ Environments and staff culture are important considerations prior to implementing evidence-based guidelines. ${ }^{48}$ Electronic reminders and tools to translate guidelines into practice are additional strategies, but further work is needed in this area. ${ }^{49}$

\section{HEALTHCARE SYSTEMS}

Healthcare systems around the world can interfere with quality pain management. Laws regarding who can prescribe opioids, which opioids are available, and access issues, especially for rural populations can significantly influence care. One of the most important systems issues is opioid availability, which is further discussed below.

\section{Opioid Availability}

Opioids are the mainstay of pain management, and yet opioids are not widely available in many countries. Opioids are usually regulated by each country's government, often the Ministry of Health $(\mathrm{MOH})$. Historical problems with opioid addiction, other fears, and current use influence the amounts of opioids allotted in each country. Economics play an additional role. Some of the poorest countries in the world are often found to have the lowest opioid amounts per capita. ${ }^{50} \mathrm{~A}$ recent analysis of global morphine consumption found significant disparity between high and low-income countries. Overall, 21\% of the world's population (high income) consumed $92 \%$ of the total global morphine. And yet the majority of cancer deaths $(70 \%)$ occur in low to middle income countries (LMICs), ${ }^{51,52}$ demonstrating a desperate need to increase opioid availability and pain management efforts in these countries. However, richer nations can also have restrictions on opioids, and so each country should be individually assessed. Figure 1 includes opioid availability comparisons for a variety of countries around the world. To note is the high consumption of Western countries versus those in other parts of the world. Turkey has recently opened a morphine production plant, hoping to increase the availability of opioids in Middle East. Nurses and other health care professionals are meeting with their $\mathrm{MOH}$ to try and increase opioids in their respective regions in order to improve pain management in palliative care patients.

\section{SUMMARY}

A number of challenges interfere with quality pain management for palliative care patients. Understanding the holistic experience of pain is the first step in addressing the physical, emotional, social, spiritual, and cultural components of the pain experience. Once holistic total pain is embraced, addressing barriers is imperative to improve pain management efforts. Patient and caregiver related barriers, clinician barriers, and systems barriers.

\section{REFERENCES}

1. World Health Organization. Palliative Care. 2017; http:// www.who.int/cancer/palliative/definition/en/. Accessed January 9, 2017, 2017.

2. Brant J. Strategies to manage pain in palliative care. In: O'Connor M, Lee S, Aranda S, eds. Palliative Care Nursing: A Guide to Practice, $3^{\text {rd }}$ ed. Victoria, Australia: Ausmed; 2012: 93-113.

3. International Association for the Study of Pain. Definition of Pain. 2014.

4. Reyes-Gibby CC, Aday LA, Anderson KO, Mendoza TR, Cleeland CS. Pain, depression, and fatigue in community-dwelling adults with and without a history of cancer. J Pain Symptom Manage. 2006; 32(2): 118-128. doi: 10.1016/j.jpainsymman.2006.01.008

5. Reyes-Gibby CC, Swartz MD, Yu X, et al. Symptom clusters of pain, depressed mood, and fatigue in lung cancer: Assessing the role of cytokine genes. Support Care Cancer. 2013; 21(11): 3117-3125. doi: 10.1007/s00520-013-1885-5

6. Dong ST, Butow PN, Costa DS, Lovell MR, Agar M. Symptom clusters in patients with advanced cancer: A systematic review of observational studies. J Pain Symptom Manage. 2014; 48(3): 411-450. doi: 10.1016/j.jpainsymman.2013.10.027

7. Yennurajalingam S, Kwon JH, Urbauer DL, Hui D, ReyesGibby CC, Bruera E. Consistency of symptom clusters among 
advanced cancer patients seen at an outpatient supportive care clinic in a tertiary cancer center. Palliat Support Care. 2013; 11(6): 473-480. doi: 10.1017/S1478951512000879

8. Syrjala KL, Jensen MP, Mendoza ME, Yi JC, Fisher HM, Keefe FJ. Psychological and behavioral approaches to cancer pain management. J Clin Oncol. 2014; 32(16): 1703-1711. doi: $10.1200 /$ jco. 2013.54 .4825

9. Laird BJ, Boyd AC, Colvin LA, Fallon MT. Are cancer pain and depression interdependent? A systematic review. Psychooncology. 2009; 18(5): 459-464. doi: 10.1002/pon.1431

10. Kwon JH, Oh SY, Chisholm G, et al. Predictors of high score patient-reported barriers to controlling cancer pain: A preliminary report. Support Care Cancer. 2013; 21(4): 1175-1183. doi: 10.1007/s00520-012-1646-x

11. National Comprehensive Cancer Network. Distress management. 2014; http://www.nccn.org/professionals/physician_gls/ pdf/distress.pdf. Accessed May 15, 2017, 2017.

12. Maher NG, Britton B, Hoffman GR. Early screening in patients with head and neck cancer identified high levels of pain and distress. J Oral Maxillofac Surg. 2013;71(8):1458-1464.

13. Lin S, Chen Y, Yang L, Zhou J. Pain, fatigue, disturbed sleep and distress comprised a symptom cluster that related to quality of life and functional status of lung cancer surgery patients. $J$ Clin Nurs. 2013; 22(9-10): 1281-1290. doi: 10.1111/jocn. 12228

14. Waller A, Groff SL, Hagen N, Bultz BD, Carlson LE. Characterizing distress, the $6^{\text {th }}$ vital sign, in an oncology pain clinic. Curr Oncol. 2012; 19(2): e53-e59. doi: 10.3747/co.19.882

15. Schwabish SD. Cognitive adaptation theory as a means to PTSD reduction among cancer pain patients. J Psychosoc Oncol. 2011; 29(2): 141-156.

16. Badr H, Gupta V, Sikora A, Posner M. Psychological distress in patients and caregivers over the course of radiotherapy for head and neck cancer. Oral Oncol. 2014; 50(10): 1005-1011 doi: 10.1016/j.oraloncology.2014.07.003

17. Carlson LE, Waller A, Groff SL, Giese-Davis J, Bultz BD. What goes up does not always come down: Patterns of distress, physical and psychosocial morbidity in people with cancer over a one year period. Psychooncology. 2013; 22(1): 168-176. doi: 10.1002/pon.2068

18. Leong M, Olnick S, Akmal T, Copenhaver A, Razzak R. How Islam influences end-of-life care: Education for palliative care clinicians. J Pain Symptom Manage. 2016; 52(6): 771-774 e773. doi: 10.1016/j.jpainsymman.2016.05.034

19. Rawdin B, Evans C, Rabow MW. The relationships among hope, pain, psychological distress, and spiritual well-being in oncology outpatients. J Palliat Med. 2013;16(2): 167-172. doi: 10.1089/jpm.2012.0223

20. Kwok W, Bhuvanakrishna T. The relationship between ethnicity and the pain experience of cancer patients: A systematic review. Indian Journal of Palliative Care. 2014; 20(3): 194-200.

21. Rahim-Williams B. Beliefs, behaviors, and modifications of type 2 diabetes self-management among African American women. J Natl Med Assoc. 2011; 103(3): 203-215. doi: 10.1016/ S0027-9684(15)30300-X

22. Chen $\mathrm{CH}$, Tang ST, Chen $\mathrm{CH}$. Meta-analysis of cultural differences in Western and Asian patient-perceived barriers to managing cancer pain. Palliat Med. 2012; 26(3): 206-221. doi: $10.1177 / 0269216311402711$

23. Im EO, Ho TH, Brown A, Chee W. Acculturation and the cancer pain experience. J Transcult Nurs. 2009; 20(4): 358-370. doi: $10.1177 / 1043659609334932$

24. Rahim-Williams B, Riley JL $3^{\text {rd }}$, Williams AK, Fillingim RB. A quantitative review of ethnic group differences in experimental pain response: Do biology, psychology, and culture matter? Pain Med. 2012; 13(4): 522-540. doi: 10.1111/j.15264637.2012.01336.x

25. Kumar P, Casarett D, Corcoran A, et al. Utilization of supportive and palliative care services among oncology outpatients at one academic cancer center: Determinants of use and barriers to access. J Palliat Med. 2012; 15(8): 923-930. doi: 10.1089/ jpm.2011.0217

26. Luckett T, Davidson PM, Boyle F, et al. Australian survey of current practice and guideline use in adult cancer pain assessment and management: Perspectives of oncologists. Asia Pac J Clin Oncol. 2012; 10(2): e99-e107. doi: 10.1111/ajco.12040

27. Silbermann M. Opioids in middle eastern populations. Asian Pac J Cancer Prev. 2010; 11(Suppl 1): 1-5.

28. Breuer B, Fleishman SB, Cruciani RA, Portenoy RK. Medical oncologists' attitudes and practice in cancer pain management: A national survey. J Clin Oncol. 2011;29(36): 4769-4775. doi: $10.1200 /$ JCO.2011.35.0561

29. Silbermann M, Pitsillides B, Al-Alfi N, et al. Multidisciplinary care team for cancer patients and its implementation in several Middle Eastern countries. Ann Oncol. 2013; 24(Suppl 7): vii41-vii47. doi: 10.1093/annonc/mdt265

30. Bennett MI, Bagnall AM, Closs SJ. How effective are patient-based educational interventions in the management of cancer pain? Systematic review and meta-analysis. Pain. 2009; 143(3): 192-199. doi: 10.1016/j.pain.2009.01.016 
31. Cummings GG, Olivo SA, Biondo PD, et al. Effectiveness of knowledge translation interventions to improve cancer pain management. J Pain Symptom Manage. 2011; 41(5): 915-939. doi: 10.1016/j.jpainsymman.2010.07.017

32. Borneman T, Koczywas M, Sun VC, Piper BF, Uman G, Ferrell B. Reducing patient barriers to pain and fatigue management. J Pain Symptom Manage. 2010; 39(3): 486-501. doi: 10.1016/j.jpainsymman.2009.08.007

33. Falvo DR. Effective Patient Education: A Guide to Increased Adherence. Sudbury, MA: Jones \& Bartlett; 2011.

34. Bennett MI, Flemming K, Closs SJ. Education in cancer pain management. Curr Opin Support Palliat Care. 2011; 5(1): 2024. doi: 10.1097/SPC.0b013e328342c607

35. Miaskowski C, Dodd MJ, West C, et al. Lack of adherence with the analgesic regimen: A significant barrier to effective cancer pain management. J Clin Oncol. 2001; 19(23): 4275-4279. doi: 10.1200/JCO.2001.19.23.4275

36. Yoong J, Traeger LN, Gallagher ER, Pirl WF, Greer JA, Temel JS. A pilot study to investigate adherence to long-acting opioids among patients with advanced lung cancer. $J$ Palliat Med. 2013; 16(4): 391-396. doi: 10.1089/jpm.2012.0400

37. Meghani SH, Bruner DW. A pilot study to identify correlates of intentional versus unintentional nonadherence to analgesic treatment for cancer pain. Pain Manag Nurs. 2013; 14(2): e22e30. doi: 10.1016/j.pmn.2011.03.003

38. McCorkle R, Ercolano E, Lazenby M, et al. Self-management: Enabling and empowering patients living with cancer as a chronic illness. CA Cancer J Clin. 2011; 61(1): 50-62. doi: 10.3322/caac. 20093

39. Beck SL, Towsley GL, Berry PH, Brant JM, Smith EM. Measuring the quality of care related to pain management: A multiple-method approach to instrument development. Nurs Res. 2010; 59(2): 85-92. doi: 10.1097/NNR.0b013e3181d1a732

40. Rustoen T, Gaardsrud T, Leegaard M, Wahl AK. Nursing pain management--a qualitative interview study of patients with pain, hospitalized for cancer treatment. Pain Manag Nurs. 2009; 10(1): 48-55. doi: 10.1016/j.pmn.2008.09.003

41. American Pain Society. Principles of Analgesic Use in the Treatment of Acute Pain and Cancer Pain. $6^{\text {th }}$ ed. Glenview, IL, USA: APS Press; 2008.

42. Federation of State Medical Boards of the United States. Model guidelines for the use of controlled substances for the treatment of pain. In: Boards FoSM, ed. Euless, TX, USA: Federation of State Medical Boards; 2013.

43. Gustafsson M, Borglin G. Can a theory-based educational intervention change nurses' knowledge and attitudes concerning cancer pain management? A quasi-experimental design. BMC Health Serv Res. 2013; 13: 328. doi: 10.1186/1472-6963-13-328

44. National Comprehensive Cancer Network. Adult Cancer Pain. NCCN Clinical Practice Guidelines in Oncology 2016; http://www.nccn.org/professionals/physician_gls/PDF/pain.pdf. Accessed February 25, 2016.

45. Caraceni A. The EPCRC project to revise the European Association for Palliative Care (EAPC) guidelines on the use of opioids for cancer pain. Palliat Med. 2011; 25(5): 389-390. doi: $10.1177 / 0269216310395871$

46. Lovell M, Agar M, Luckett T, Davidson PM, Green A, Clayton J. Australian survey of current practice and guideline use in adult cancer pain assessment and management: Perspectives of palliative care physicians. J Palliat Med. 2013;16(11):14031409. doi: 10.1089/jpm.2013.0245

47. Dulko D, Hertz E, Julien J, Beck S, Mooney K. Implementation of cancer pain guidelines by acute care nurse practitioners using an audit and feedback strategy. J Am Acad Nurse Pract. 2010; 22(1): 45-55. doi: 10.1111/j.1745-7599.2009.00469.x

48. Sanders S, Mackin ML, Reyes J, et al. Implementing evidence-based practices: Considerations for the hospice setting. Am J Hosp Palliat Care. 2010; 27(6): 369-376. doi: 10.1177/1049909109358695

49. Fine $\mathrm{P}$, Herr K, Titler M, et al. The cancer pain practice index: A measure of evidence-based practice adherence for cancer pain management in older adults in hospice care. J Pain Symptom Manage. 2010; 39(5): 791-802. doi: 10.1016/j.jpainsymman.2009.09.027

50. World Report. Human Rights Watch, 2011 Web site. https:// www.hrw.org/world-report/2011.

51. Berterame S, Erthal J, Thomas J, et al. Use of and barriers to access to opioid analgesics: A worldwide, regional, and national study. Lancet. 2016; 387(10028): 1644-1656. doi: 10.1016/ S0140-6736(16)00161-6

52. International Narcotics Control Board. Availability of narcotic drugs for medical use. 2017; https://www.incb.org/incb/en/ narcotic-drugs/Availability/availability.html. Accessed February 16, 2017, 2017. 